\title{
Role Ordering (RO) Scheduler for Distributed Objects
}

\author{
TOMOYA ENOKIDO ${ }^{\dagger}$
}

\begin{abstract}
A role-based access control model is used to make a system secure. A role concept shows a job function in an enterprise. Traditional locking protocols and timestamp ordering schedulers are based on principles "first-comer-winner" and "timestamp order" to make multiple conflicting transactions serializable, respectively. In this paper, we discuss a concurrency control based on the significancy of roles assigned to transactions. We define a significantly dominant relation on roles. We discuss a role ordering (RO) scheduler based on the role concept. We evaluate the RO scheduler compared with the two-phase locking (2PL) protocol.
\end{abstract}

\section{Introduction}

Information systems like database systems ${ }^{10), 13)}$ adopt role-based access control (RBAC) models ${ }^{3), 6), 9), 12), 15)}$ to make the systems secure. A role shows a job function like president and secretary, which each person performs in an enterprise. We use word "role" in software engineering field. A role is a set of access rights. An access right is realized in a pair $\langle o, o p\rangle$ of an object $o$ and a method $o p$. Only if a role $R$ which includes an access right $\langle o, o p\rangle$ is granted to a subject, the subject is allowed to manipulate the object $o$ through the method op. In the discretionary approach ${ }^{10), 13)}$, a subject who is granted a role can further grant the role to another subject.

A transaction is an atomic sequence of methods which are performed on objects ${ }^{1), 7)}$. A pair of methods conflict if and only if (iff) the result obtained by performing the methods depends on the computation order. A pair of transactions are referred to as conflict if the transactions manipulate a same object through conflicting methods. A collection of conflicting transactions are required to be serializable in order to keep objects mutually consistent. In order to realize the serializability of multiple conflicting transactions, locking protocols ${ }^{1), 7)}$ are widely used. Locking protocols are based on a principle that only the first comer is a winner and the others are losers. Another way is a timestamp ordering (TO) scheduler ${ }^{1)}$. By using the TO scheduler, transactions are totally ordered in their timestamps.

In this paper, we discuss a role ordering $(R O)$ scheduler to make a set of transactions

$\dagger$ Faculty of Business Administration, Rissho University serializable based on roles associated for transactions. Each job is realized to be a collection of transactions in an enterprise. Let $T_{1}$ and $T_{2}$ be a pair of transactions which are associated with roles $R_{1}$ and $R_{2}$, respectively, and which manipulate an object $o$ in a conflicting manner. Here, the transaction $T_{1}$ manipulates the object $o$ before $T_{2}$ if the role $R_{1}$ is more significant than the other role $R_{2}$, i.e. a job function shown by $R_{1}$ is more significant than $R_{2}$ in an enterprise. This means the more significant job a transaction does, the earlier an object can be manipulated by the transaction. In the RO scheduler, conflicting methods issued by transactions are ordered in the significancy of the roles. Transactions can concurrently manipulate objects in such an order that persons really do their jobs in an enterprise.

In Section 2, we present a system model. In Section 3, we define significantly dominant relations among roles. In Section 4, we discuss the role ordering (RO) serializability. In Section 5, we discuss the RO scheduler. In Section 6, we evaluate the RO scheduler compared with the two-phase locking (2PL) protocol.

\section{System Model}

\subsection{Object-based System}

An object-based system is composed of objects ${ }^{8)}$ distributed in networks. An object is an encapsulation of data and methods for manipulating the data. A method is more abstract than primitive methods like read and write. A pair of methods $o p_{1}$ and $o p_{2}$ conflict $\left(o p_{1}\right.$ $\left.\diamond o p_{2}\right)$ iff the result obtained by performing

The initial version of this paper was presented at the DPS workshop (DPSWS12) held on Dec. 2004, which was sponsored by SIGDPS. This paper was recommended to be submitted to IPSJ Journal by the program chair of DPSWS12. 
the methods depends on the computation order. Otherwise, a pair of the methods $o p_{1}$ and $o p_{2}$ are compatible $\left(o p_{1} \square o p_{2}\right)$.

A transaction is an atomic sequence of methods ${ }^{1)}$. Multiple transactions are concurrently performed on objects. Multiple conflicting transactions are required to be serializable to keep objects mutually consistent ${ }^{1), 7)}$. Let $T_{i}$ be a transaction which issues a method $o p_{1 i}$ to an object $o_{1}$. Suppose there are a pair of transactions $T_{1}$ and $T_{2}$ where $o p_{11}$ and $o p_{12}$ conflict on the object $o_{1}$ as well as the methods $o p_{21}$ and $o p_{22}$ on the object $o_{2}$. If the method $o p_{11}$ is performed on the object $o_{1}$ before $o p_{21}, o p_{21}$ is required to be performed before $o p_{22}$ on the other object $o_{2}$ according to the serializebility theory ${ }^{1)}$. Let $\mathbf{T}$ be a set of transactions $\left\{T_{1}\right.$, ..., $\left.T_{n}\right\}$. Let $H$ be a schedule of transactions in $\mathbf{T}$, i.e., sequence of methods performed. A transaction $T_{i}$ precedes another transaction $T_{j}$ $\left(T_{i} \rightarrow_{H} T_{j}\right)$ in $H$ iff a method $o p_{i}$ from $T_{i}$ is performed before a method $o p_{j}$ from $T_{j}$ where $o p_{i}$ $\diamond o p_{j}$. A schedule $H$ is serializable iff the precedent relation $\rightarrow_{H}$ is acyclic. In the timestamp ordering (TO) scheduler ${ }^{1)}$, each transaction $T_{i}$ is assigned with time $t s\left(T_{i}\right)$ showing what time the transaction $T_{i}$ is initiated on a client. A pair of conflicting methods issued by transactions $T_{1}$ and $T_{2}$ are performed in the timestamp order. In the two-phase locking (2PL) proto$\mathrm{col}^{7)}$, the transaction $T_{1}$ is performed if a pair of the objects $o_{1}$ and $o_{2}$ are locked before the other transaction $T_{2}$. The transaction $T_{2}$ cannot manipulate the objects $o_{1}$ and $o_{2}$ until the transaction $T_{1}$ releases the objects.

\subsection{Roles}

In access control models 1),2),4),6),11),12),14),15), a system is composed of two types of entities, subject and object. A subject manipulates an object. A role shows a job function in an enterprise like president. Each subject $s$ plays a role. A subject which plays a more significant role should be more prioritized than less significant subjects. A task is realized as a transaction. If a pair of tasks in different jobs use an object, one task in a more significant job should take the object earlier than the other.

A role is a collection of access rights in a rolebased access control (RBAC) model ${ }^{12)}$. An access right is a pair $\langle o, o p\rangle$ of an object $o$ and a method op. A subject $s$ is first granted a role $R$. Then, the subject can issue an access request op to an object $o$ only if an access right $\langle o, o p\rangle$ is included in $R$. We assume each transaction is

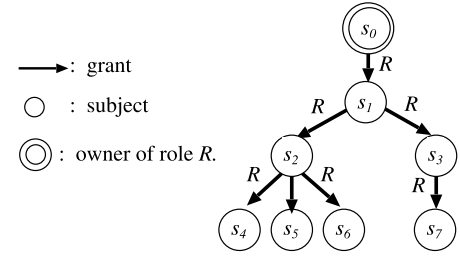

Fig. 1 Discretionary approach.

associated with only one role in this paper. Let $\operatorname{subject}(T)$ denote a subject which initiates a transaction $T$. Let $\operatorname{role}(T)$ show a role which is associated to a transaction $T$.

\section{Significancy on Roles}

\subsection{Significancy of Subjects on a Role}

The relational database systems take the discretionary approach ${ }^{10), 13)}$. We take the discretionary approach to adopting the role-based access control (RBAC) model ${ }^{12)}$ to objectbased systems. First, suppose that a subject $s_{0}$ creates a role $R$. Here, the subject $s_{0}$ is an owner of the role $R$, denoted by owner $(R)$. Then, the subject $s_{0}$ grants the role $R$ to another subject $s_{1}$. The subject $s_{1}$ furthermore grants the role $R$ to a pair of subjects $s_{2}$ and $s_{3}$ (Fig. 1). If the subject $s_{1}$ changes the role $R$, the role $R$ granted to the subjects $s_{0}$ and $s_{2}$ is also changed.

We define a precedent relation among subjects showing which subjects are more significant than others with respect to a role $R$ :

- A subject $s_{1}$ is more significant than another subject $s_{2}$ with respect to a role $R$ $\left(s_{1} \succ_{R} s_{2}\right)$ if and only if (iff) the subject $s_{1}$ grants the role $R$ to the subject $s_{2}$ or $s_{1}$ $\succ_{R} s_{3} \succ_{R} s_{2}$ for some subject $s_{3}$.

A pair of subjects $s_{1}$ and $s_{2}$ are independent with respect to a role $R\left(s_{1} \|_{R} s_{2}\right)$ iff $s_{1}$ and $s_{2}$ are granted the role $R$ and neither $s_{1} \succ_{R} s_{2}$ nor $s_{2} \succ_{R} s_{1}$. Because, there are no significant relations with respect to a role $\mathrm{R}\left(\succ_{R}\right)$ which is defined in above between subjects $s_{1}$ and $s_{2}$.

\subsection{Significancy of Roles}

Next, we discuss which roles are more significant than other roles. There are two types of methods, class and object methods for a class. Class methods are ones for creating and dropping an object for the class. On the other hand, object methods are ones for manipulating an object of the class. There are two types of object methods, change and output types. An output type of method is a method for de- 
riving data from an object. On the other hand, a change type of method is one for changing a state of an object.

Let us consider a pair of change methods withdraw and deposit on a bank object. In our life, a subject more carefully issues a method withdraw than a method deposit because the account value in the bank object is decremented through withdraw. This example shows that some methods are considered to be more significant than other methods by an application. Here, a method withdraw is referred to as more semantically significant than another method deposit (withdraw $\succ$ deposit). A method $o p_{1}$ is referred to as semantically significantly equivalent with another method $o p_{2}\left(o p_{1} \cong\right.$ $o p_{2}$ ) iff neither $o p_{1} \succ o p_{2}$ nor $o p_{2} \succ o p_{1}$. A method $o p_{1}$ semantically significantly dominates a method $o p_{2}\left(o p_{1} \succeq o p_{2}\right)$ iff $o p_{1} \succ o p_{2}$ or $o p_{1} \cong o p_{2}$.

[Definition] A method $o p_{1}$ is more significant than another method $o p_{2}\left(o p_{1} \succ o p_{2}\right)$ iff one of the following conditions is satisfied:

1. $o p_{1}$ is a class type and $o p_{2}$ is an object type.

2. $o p_{1}$ and $o p_{2}$ are an object type where $o p_{1}$ is a change type and $o p_{2}$ is just an output one.

3. Object types of methods $o p_{1}$ and $o p_{2}$ are same types and $o p_{1} \succ o p_{2}$.

A method $o p_{1}$ is significantly equivalent with another method $o p_{2}\left(o p_{1} \equiv o p_{2}\right)$ iff neither $o p_{1} \succ o p_{2}$ nor $o p_{2} \succ o p_{1}$. A method $o p_{1}$ significantly dominates another method $o p_{2}$ $\left(o p_{1} \succeq o p_{2}\right)$ iff $o p_{1} \succ o p_{2}$ or $o p_{1} \equiv o p_{2}$.

Objects are classified into some security classes ${ }^{4), 5)}$. An object $o_{1}$ is more significant than another object $o_{2}\left(o_{1} \succ o_{2}\right)$ if $o_{1}$ is more secure than $o_{2}$. A pair of objects $o_{1}$ and $o_{2}$ are significantly equivalent $\left(o_{1} \equiv o_{2}\right)$ if neither $o_{1}$ $\succ o_{2}$ nor $o_{2} \prec o_{1}$. An object $o_{1}$ significantly dominates another object $o_{2}\left(o_{1} \succeq o_{2}\right)$ iff $o_{1} \succ$ $o_{2}$ or $o_{1} \equiv o_{2}$.

Next, we discuss which access right $\left\langle o_{1}, o p_{1}\right\rangle$ or $\left\langle o_{2}, o p_{2}\right\rangle$ is more significant than the other based on the significantly dominant relation $\succeq$ of methods.

[Definition] An access right $\left\langle o_{1}, o p_{1}\right\rangle$ is more significant than another access right $\left\langle o_{2}, o p_{2}\right\rangle$ $\left(\left\langle o_{1}, o p_{1}\right\rangle \succ\left\langle o_{2}, o p_{2}\right\rangle\right)$ iff 1$) o_{1} \succ o_{2}$ or 2$) o p_{1} \succ$ $o p_{2}$ if $o_{1} \equiv o_{2}$.

A pair of access rights $\left\langle o_{1}, o p_{1}\right\rangle$ and $\left\langle o_{2}, o p_{2}\right\rangle$ are significantly equivalent $\left(\left\langle o_{1}, o p_{1}\right\rangle \equiv\right.$ $\left.\left\langle o_{2}, o p_{2}\right\rangle\right)$ iff neither $\left\langle o_{1}, o p_{1}\right\rangle \succ\left\langle o_{2}, o p_{2}\right\rangle$ nor $\left\langle o_{1}, o p_{1}\right\rangle \prec\left\langle o_{2}, o p_{2}\right\rangle$. An access right $\left\langle o_{1}, o p_{1}\right\rangle$ significantly dominates another access right $\left\langle o_{2}, o p_{2}\right\rangle\left(\left\langle o_{1}, o p_{1}\right\rangle \succeq\left\langle o_{2}, o p_{2}\right\rangle\right)$ iff $\left\langle o_{1}, o p_{1}\right\rangle \succ$ $\left\langle o_{2}, o p_{2}\right\rangle$ or $\left\langle o_{1}, o p_{1}\right\rangle \equiv\left\langle o_{2}, o p_{2}\right\rangle$.

Finally, we discuss which role is more significant than another role based on the significantly dominant relation $\succeq$ of access rights.

[Definition] A role $R_{1}$ significantly dominates another role $R_{2}\left(R_{1} \succeq R_{2}\right)$ if for every access right $\left\langle o_{2}, o p_{2}\right\rangle$ in $R_{2}$, there is at least one access right $\left\langle o_{1}, o p_{1}\right\rangle$ in $R_{1}$ such that $\left\langle o_{1}, o p_{1}\right\rangle$ $\succeq\left\langle o_{2}, o p_{2}\right\rangle$ and no access right $\left\langle o_{3}, o p_{3}\right\rangle$ in $R_{2}$ such that $\left\langle o_{3}, o p_{3}\right\rangle \succeq\left\langle o_{1}, o p_{1}\right\rangle$.

A role $R_{1}$ is significantly equivalent with another role $R_{2}\left(R_{1} \equiv R_{2}\right)$ if $R_{1} \succeq R_{2}$ and $R_{2}$ $\succeq R_{1}$. A least upper bound (lub) $R_{1} \cup R_{2}$ of roles $R_{1}$ and $R_{2}$ is a role $R_{3}$ such that $R_{3} \succeq R_{1}$ and $R_{3} \succeq R_{2}$ and there is no role $R_{4}$ such that $R_{3} \succeq R_{4} \succeq R_{1}$ and $R_{3} \succeq R_{4} \succeq R_{2}$. A greatest lower bound ( $g l b) R_{1} \cap R_{2}$ is similarly defined.

\section{Serializability}

Let $\mathbf{T}$ be a set of transactions which are being performed in a system. We define which transaction $T_{1}$ or $T_{2}$ in $\mathbf{T}$ is significant based on the significantly dominant relations of subjects and roles.

[Definition] A transaction $T_{1}$ significantly dominates another transaction $T_{2}\left(T_{1} \succeq T_{2}\right)$ iff $\operatorname{role}\left(T_{1}\right) \succeq \operatorname{role}\left(T_{2}\right)$ or $\operatorname{subject}\left(T_{1}\right) \succ_{R}$ $\operatorname{subject}\left(T_{2}\right)$ if $\operatorname{role}\left(T_{1}\right)=\operatorname{role}\left(T_{2}\right)=R$.

A transaction $T_{1}$ is significantly equivalent with another transaction $T_{2}\left(T_{1} \equiv T_{2}\right)$ if $T_{1} \succeq$ $T_{2}$ and $T_{2} \succeq T_{1}$. A least upper bound $(l u b) T_{1}$ $\bigcup T_{2}$ of transactions $T_{1}$ and $T_{2}$ is a transaction $T_{3}$ where $T_{3} \succeq T_{1}$ and $T_{3} \succeq T_{2}$ and there is no transaction $T_{4}$ such that $T_{3} \succeq T_{4} \succeq T_{1}$ and $T_{3}$ $\succeq T_{4} \succeq T_{2}$. A greatest lower bound (glb) $T_{1} \bigcap$ $T_{2}$ is defined similarly. We assume that a top transaction $T$ and a bottom transaction $\perp$ exist where $T \succeq T \succeq \perp$ for every transaction $T$.

A schedule $H$ is an execution sequence of methods from transactions in $\mathbf{T}$. A transaction $T_{1}$ precedes another transaction $T_{2}$ in the schedule $H\left(T_{1} \rightarrow_{H} T_{2}\right)$ iff a method $o p_{1}$ from $T_{1}$ is performed before a method $o p_{2}$ from $T_{2}$ which conflicts with $o p_{1}$. A schedule $H$ is serializable iff the precedent relation $\rightarrow_{H}$ is acyclic according to the traditional theory ${ }^{1)}$. A schedule $H$ of a transaction set $\mathbf{T}$ is shown in a partially ordered set $\left\langle\mathbf{T}, \rightarrow_{H}\right\rangle$.

[Definition] A transaction $T_{1}$ significantly precedes another transaction $T_{2}$ in a schedule 
$H$ of a transaction set $\mathbf{T}\left(T_{1} \Rightarrow_{H} T_{2}\right)$ iff $T_{1} \rightarrow_{H}$ $T_{2}$ and $T_{1} \succeq T_{2}$, i.e. $T_{1} \Rightarrow_{H} T_{2}$ if $o p_{1}$ and $o p_{2}$ conflict and $o p_{1}$ is performed before $o p_{2}$ for every pair of methods $o p_{1}$ and $o p_{2}$ from $T_{1}$ and $T_{2}$, respectively.

Suppose a transaction $T_{1}$ precedes another transaction $T_{2}$ in a schedule $H$. Here, if $T_{1}$ $\succeq T_{2}$, a precedent relation " $T_{1} \rightarrow_{H} T_{2}$ " is legal in a schedule $H$. On the other hand, if $T_{1} \prec$ $T_{2}$, " $T_{1} \rightarrow_{H} T_{2}$ " is illegal in a schedule $H$. A schedule $H=\left\langle\mathbf{T}, \rightarrow_{H}\right\rangle$ is legal iff $T_{1} \rightarrow_{H} T_{2}$ if $T_{1} \succeq T_{2}$ for every pair of transactions $T_{1}$ and $T_{2}$ in a schedule $H$ in $\mathbf{T}$. This means, for every pair of conflicting methods $o p_{1}$ and $o p_{2}$ from transactions $T_{1}$ and $T_{2}$ where $T_{1} \succeq T_{2}, o p_{1}$ is performed before $o p_{2}$.

In order to make a schedule legal, methods from transactions are required to be buffered until all the transactions are initiated. However, the throughput of the system is degraded. In order to increase the throughput, only some number of transactions in $\mathbf{T}$ which are initiated during some time units are scheduled.

A schedule $H=\left\langle\mathbf{T}, \rightarrow_{H}\right\rangle$ is partitioned into subschedules $H_{1}, \ldots, H_{m}$ where each subschedule $H_{i}=\left\langle\mathbf{T}_{i}, \rightarrow_{H_{i}}\right\rangle(i=1, \ldots, m)$ satisfies the following conditions:

[Role ordering (RO) partition conditions]

1. $\mathbf{T}_{i} \cap \mathbf{T}_{j}=\phi$ for every pair of subschedules $H_{i}$ and $H_{j}$ and $\mathbf{T}_{1} \cup \cdots \cup \mathbf{T}_{n}=\mathbf{T}$. That is, every pair of $T_{i}$ and $T_{j}$ in $\mathbf{T}$ are independent.

2. A precedent relation $T_{1} \rightarrow_{H} T_{2}$ is legal in a schedule $H$ if $T_{1} \rightarrow H_{i} T_{2}$ for every pair of transactions $T_{1}$ and $T_{2}$ in $\mathbf{T}_{i}$ of a subschedule $H_{i}$.

3. For every pair of subschedules $H_{i}$ and $H_{j}$, if $T_{i 1} \rightarrow_{H} T_{j 1}$ for some pair of transactions $T_{i 1}$ in $H_{i}$ and $T_{j 1}$ in $H_{j}$, there are no pair of transactions $T_{i 2}$ in $H_{i}$ and $T_{j 2}$ in $H_{j}$ such that $T_{j 2} \rightarrow_{H} T_{i 2}$.

A role is assigned to each transaction when the subject initiates the transaction. Significantly dominant relations among transactions are defined based on the significancy of roles assigned to each transaction, which we defined at the beginning of this section. Here, suppose that six transactions are initiated (see Fig. 2) and a transaction $T_{1}$ significantly dominates a transaction $T_{2}\left(T_{1} \succeq T_{2}\right), T_{3} \succeq T_{2}, T_{4} \succeq T_{5}$, $T_{4} \succeq T_{6}, T_{4} \succeq T_{2}$, and $T_{6} \succeq T_{3}$. In addition, suppose that a schedule $H$ is RO partitioned into a pair of subschedules $H_{1}$ with $\mathbf{T}_{1}=\left\{T_{1}\right.$,

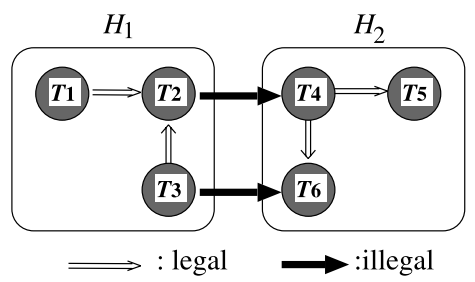

Fig. 2 Schedule $H$.

$\left.T_{2}, T_{3}\right\}$ and $H_{2}$ with $\mathbf{T}_{2}=\left\{T_{4}, T_{5}, T_{6}\right\}$. If the subschedule $\mathrm{H}_{2}$ is started before another subschedule $H_{1}$ is not completed, a pair of transactions $T_{2}$ and $T_{4}$ are concurrently performed and $T_{2}$ may be completed before $T_{4}\left(T_{2} \rightarrow_{H}\right.$ $T_{4}$ ) although $T_{2} \preceq T_{4}$. Similarly, $T_{3}$ may be completed before $T_{6}\left(T_{3} \rightarrow_{H} T_{6}\right)$ although $T_{3}$ $\preceq T_{6}$. Therefore, the schedule $H$ may become illegal. In this case, since $T_{2} \preceq T_{4}$ and $T_{3} \preceq$ $T_{6}$, the transactions $T_{4}$ and $T_{6}$ cannot be performed as long as every transaction completes in the subschedule $H_{1}$.

[Definition] Let $\mathbf{T}$ be a set of transactions. A history $H$ of $\mathbf{T}$ is $R O$ serializable iff the schedule $H$ is RO partitioned.

It is straightforward for the following theorem to hold.

[Theorem] A history $H$ is serializable if $H$ is RO serializable.

\section{Role-Ordering (RO) Scheduler}

\subsection{One-object Model}

We discuss a role-ordering (RO) scheduler for a single object. Multiple transactions on clients issue methods to an object $o$. A transaction lastly issues a commit (c) or abort (a) method. An RO scheduler is composed of a receipt queue $R Q$ and an auxiliary receipt queue $A R Q$. Let $\operatorname{Tr}(o p)$ show a transaction which issues a method op. The following procedures are supported to manipulate a queue $Q$ :

1. enqueue $(o p, Q)$ : a method $o p$ is enqueued into a queue $Q$.

2. op $:=\operatorname{dequeue}(Q)$ : a method $o p$ is dequeued from a queue $Q$.

3. $o p:=\operatorname{top}(Q)$ : a method op is a top method in a queue $Q$.

4. $\operatorname{ROsort}(Q)$ : all methods in a queue $Q$ are sorted in the significantly dominant relation $\succeq$ of transactions.

Variables $\mathbf{E}$ and $\mathbf{T E}$ show sets of methods and transactions being currently performed on an object $o$, respectively. A variable $\mathbf{C}$ denotes a transaction which is performed on the object 
$o$ and which is significantly dominated by every transaction performed. Initially, $\mathbf{C}:=\top$. There are following procedures to perform a method $o p$ on an object $o$ :

1. $\operatorname{conflict}(o p, \mathbf{E})$ : false if $\mathbf{E}=\phi$ or a method op does not conflict with every method in $\mathbf{E}$, else true.

2. $\operatorname{perform}(o p)$ : a method $o p$ is performed on the object $o$.

Suppose methods in transactions $T_{1}, \ldots, T_{m}$ are being performed, $\mathbf{T E}=\left\{T_{1}, \ldots, T_{m}\right\}$. Methods of the transactions $T_{1}, \ldots, T_{m}$ being performed are stored in the variable $\mathbf{E}$. Here, a variable $\mathbf{C}$ shows a transaction $T_{i}$ where $T_{i} \preceq$ $T_{j}$ for every $i$ and $j(i=j=1, \ldots, m)$. If $T \preceq$ $\mathbf{C}$, the method $o p$ is enqueued into the receipt queue $R Q$. However, if $T \succ \mathbf{C}, o p$ is enqueued into the auxiliary receipt queue $A R Q$.

[Delivery of a method $o p$ ]

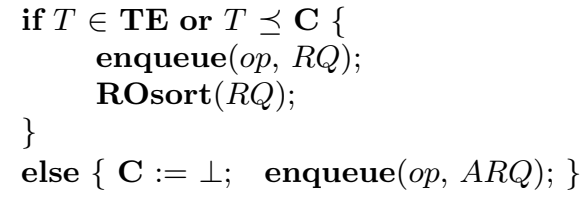

Methods in the receipt queue $R Q$ are performed on an object $o$ as follows:

[Execution of methods]

1. if $\mathbf{T E}=\phi,\{$

$\mathbf{C}:=\top$;

Every method op in $A R Q$ is moved to $R Q$; ROsort $(R Q)$;

$/{ }^{*}$ one subschedule is ended and a new schedule is started. $* /\}$

2. $o p=\operatorname{top}(R Q)$;

3. if conflict $(o p, \mathbf{E})$, return; else $\{o p:=\operatorname{dequeue}(R Q)$;

$$
\begin{aligned}
& \text { if } \operatorname{Tr}(o p) \notin \mathbf{T E}, \mathbf{T E}:=\mathbf{T E} \cup\{\operatorname{Tr}(o p)\} ; \\
& \mathbf{E}:=\mathbf{E} \cup\{o p\} ; \\
& \text { if } \operatorname{Tr}(o p) \prec \mathbf{C}, \mathbf{C}:=\operatorname{Tr}(o p) ; \\
& \text { perform }(o p) ;\}
\end{aligned}
$$

If a method op completes, the following procedure is performed:

[Completion of method $o p$ ]

1. $\mathbf{E}:=\mathbf{E}-\{o p\}$;

2. $\mathbf{T E}:=\mathbf{T E}-\{\operatorname{Tr}(o p)\}$ if $o p=c$ or $o p=a$;

3. Methods in $R Q$ are performed in the execution procedure presented here.

If a top method $o p_{1}$ conflicting with some method being performed is kept waiting in the receipt queue $R Q$, every other method in $R Q$ is required to be waited. We discuss how to improve the performance.

[Definition] A method op is ready in a receipt queue $R Q$ iff $o p$ is compatible with not only every method in being performed but also every waiting method preceding $o p$ in the receipt queue $R Q$.

We introduce the following procedures:

1. $\operatorname{ready}(o p, R Q, \mathbf{E}):$ true if a method $o p$ is ready in the receipt queue $R Q$, else false.

2. $o p_{1}:=\operatorname{next}(o p, R Q): o p_{1}$ is a method in the receipt queue $R Q$ which directly follows an method op.

Let $o p$ be a top method in $R Q$. If $o p$ conflicts with some method being performed, the following procedure is performed:

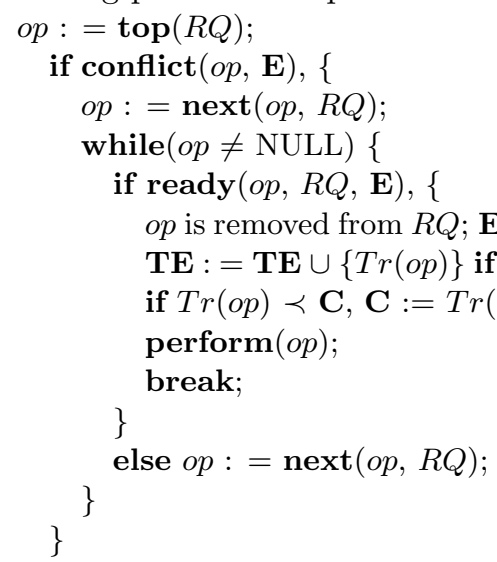

[Theorem] A schedule of a transaction set $\mathbf{T}$ obtained by the RO scheduler is ROserializable.

[Proof] A subschedule obtained from the receipt queue $R Q$ is RO subschedule. A schedule of the transaction set $\mathbf{T}$ is RO partitioned into the subsequences.

\subsection{Distributed object model}

In a distributed model, there are multiple objects $o_{1}, \ldots, o_{l}(l>1)$ distributed in multiple servers and multiple transactions $T_{1}, \ldots$, $T_{m}(m>1)$ on multiple clients $c_{1} \ldots, c_{n}(n>$ $1)$. A request is written in a pair $\left\langle o_{i}, o p_{i}\right\rangle$ of an object $o_{i}$ and a method $o p_{i}$ on the object $o_{i}$. Let $m \operatorname{set}\left(T_{t}\right)$ be a set of requests which will be issued by a transaction $T_{t}$ and $\operatorname{oset}\left(T_{t}\right)$ be a set of objects to be manipulated, $\{o \mid\langle o, o p\rangle \in$ $\left.m \operatorname{set}\left(T_{t}\right)\right\}(t=1, \ldots, m)$. Each transaction $T_{t}$ first sends $m s e t\left(T_{t}\right)$ to every object $o_{i}$ to be manipulated in $\operatorname{oset}\left(T_{t}\right)$. After sending $\operatorname{mset}\left(T_{t}\right)$, the transaction $T_{t}$ issues methods to the objects. A transaction $T_{t}$ lastly issues a issues a commit (c) or an abort (a) method to every object in oset $\left(T_{t}\right)$.

Each client $c_{s}$ has a variable $f$ which is initialized by one $(f=1)(s=1, \ldots, n)$. Each client 
$c_{s}$ periodically sends a fence message which includes the variable $f$ of $c_{s}$ to all objects in a system. After sending the fence message, the client $c_{s}$ increments the variable $f$ by one.

There are local receipt queues $R Q_{i 1}, \ldots, R Q_{i n}$ in each object $o_{i}(i=1, \ldots, l)$. Methods and $m s e t\left(T_{t}\right)$ issued from transactions $T_{t}$ on a client $c_{s}$ to an object $o_{i}$ are stored in each local receipt queue $R Q_{i s}(s=1, \ldots, n)$. We assume a communication network supports every pair of an object $o_{i}$ and a client $c_{s}$ with a reliable communication channel. Requests in local receipt queues $R Q_{i 1}, \ldots, R Q_{i n}$ are moved to a global receipt queue $G R Q_{i}$ on the object $o_{i}$. Here, requests in the queue $G R Q_{i}$ are sorted in the significantly dominant relation $\succeq$ of transactions. The following conditions have to be satisfied for a collection of global receipt queues $G R Q_{1}, \ldots$, $G R Q_{l}$ for objects $o_{1}, \ldots, o_{l}$, respectively, to realize the serializability of multiple transactions:

\section{[Role-based serializability (RBS)]}

1. Methods in every $G R Q_{i}$ are sorted in the significantly dominant relation $\succeq$ of transactions $(i=1, \ldots, l)$.

2. For a top method $o p_{t}$ from a transaction $T_{t}$ in each global receipt queue $G R Q_{i}$, if there is a method $o p_{u}^{\prime}$ from the transaction $T_{u}$ in $G R Q_{i}$ which the method $o p_{u}$ precedes and conflicts with $o p_{t}, o p_{u}^{\prime}$ precedes $o p_{t}$ in every global receipt queue $G R Q_{j}$ where $o p_{t}$ and $o p_{u}$ are methods form $T_{t}$ and $T_{u}$, respectively, and $o p_{u}^{\prime}$ and $o p_{t}$ conflict with one another.

We discuss how role ordering (RO) scheduler on each object handles methods and $\operatorname{mset}\left(T_{l}\right)$ received from multiple transactions on multiple clients in order to satisfy the RBS conditions. Each object $o_{i}$ has a variable $f_{o_{i}}$ which was initialized to be one $\left(f_{o_{i}}=1\right)$.

\section{[Receiving procedure]}

If there is a fence message $k_{s}$ whose variable $f$ is equal to a variable $f_{o_{i}}$ of the object $o_{i}(f$ $\left.=f_{o_{i}}\right)$ in every local receipt queue $R Q_{i s}$, methods and $\operatorname{mset}\left(T_{t}\right)$ preceding the fence message $k_{s}$ are dequeued from every local receipt queue $R Q_{i s}$. Then, one of the following procedures is performed (Fig. 3).

1. If a dequeued message is $\operatorname{mset}\left(T_{t}\right)$, $\operatorname{mset}\left(T_{t}\right)$ is enqueued into an auxiliary global receipt queue $\left(A G R Q_{i}\right)$ of $o_{i}$ in significantly dominant relation of transaction $T_{t}$. Here, $\operatorname{mset}\left(T_{t}\right)$ cannot be enqueued into $A G R Q_{i}$ by beyond a fence message

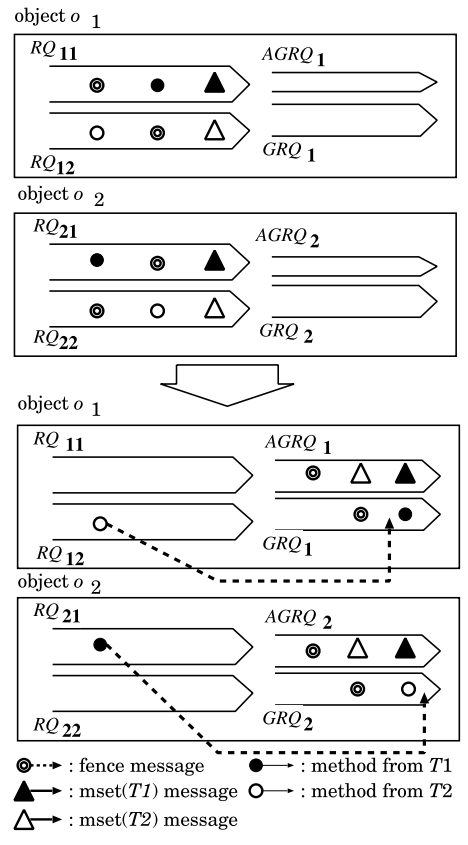

Fig. 3 State of local receipt queues.

which has already enqueued into $A G R Q_{i}$.

2. If a dequeued message is a method $o p_{i t}$ issued by a transaction $T_{t}$ to the object $o_{i}$, RO scheduler searches $A G R Q_{i}$ to confirm where the $\operatorname{mset}\left(T_{t}\right)$ of the transaction $T_{t}$ is enqueuing into. There are three cases. 1) If $m \operatorname{set}(T)$ is enqueuing between the top of $A G R Q_{i}$ and the first fence message $k_{1}$ in $A G R Q_{i}$, the method $o p_{i t}$ is stored between the top of $G R Q_{i}$ and the first fence message $k_{1}$ of $G R Q_{i}$ in the significantly dominant relation of transaction $\succeq$. 2) If $\operatorname{mset}(T)$ is enqueuing between a pair of fence messages $k_{l}$ and $k_{m}$ of $A G R Q_{i}$, the method $o p_{i t}$ is stored between a pair of fence messages $k_{l}$ and $k_{m}$ of $G R Q_{i}$ in $\succeq$. 3) If $\operatorname{mset}(T)$ is enqueuing between last fence message $k_{n}$ in $A G R Q_{i}$ and the end of $A G R Q_{i}$, the method is enqueued between last fence message $k_{n}$ of $G R Q_{i}$ and the end of $G R Q_{i}$ in $\succeq$.

3. If all top messages in every local receipt queue $R Q_{i 1}, \ldots, R Q_{i n}$ are fence message including the same value of $f$, a fence message $k$ is enqueued into $G R Q_{i}$ and $A G R Q_{i}$ of the object $o_{i}$.

Next, we discuss how role ordering (RO) scheduler on an object $o_{i}$ delivers methods from $G R Q_{i}$ to an object $o_{i}$.

\section{[Delivery procedure]}


1. If the top method of $G R Q_{i}$ is a method $o p_{i t}$ and the following two conditions are satisfied, $o p_{i t}$ is delivered to an object $o_{i}$.

1.1 The method $o p_{i t}$ does not conflict with every method which is currently performed on the object $o_{i}$.

1.2 Transactions which conflict with the transaction $T_{t}$ and precede the transaction $T_{t}$ in $A G R Q_{i}$ are completed on the object $o_{i}$.

2. If the top method of $G R Q_{i}$ is a fence message $k$, the RO scheduler waits for completion of the currently performing methods on $o_{i}$. After all methods are completed, the fence message $k$ is removed from $G R Q_{i}$.

Next, we show a behavior of each object.

\section{[Completion of a method]}

1. If a method completed on an object $o_{i}$ is commit (c) or abort (a) of a transaction $T_{t}$, RO scheduler removes the $\operatorname{mset}\left(T_{t}\right)$ of the transaction $T_{t}$ from $A G R Q_{i}$.

\section{Evaluation}

We evaluate the role ordering ( $\mathrm{RO}$ ) scheduler for a single object system in terms of computation time of each method compared with the traditional two-phase locking (2PL) protocol. In the evaluation, an object $o$ supports ten types of methods. We assume it takes a same time to perform every method. We assume one method can be performed for one time unit if there is no other transaction. The computation ratio $\tau$ is defined to be the ratio of the total number of methods effectively performed to the total processing time units. If all the transactions are serially performed, the computation ratio $\tau$ is 1.0 which is the maximum. $\tau=0$ if no method is performed. A conflicting relation on the methods is randomly defined so that each method averagely conflicts with $10 \%$ of the other methods. There are five roles $R_{1}, \ldots, R_{5}$. Each role $R_{i}$ includes three access rights, which are randomly selected out of ten possible access rights on the object $o(i=1, \ldots, 5)$. There are three subjects $s_{0}, s_{1}$, and $s_{2}$. The subject $s_{0}$ is an owner of the roles $R_{1}, \ldots, R_{5}$. The subject $s_{0}$ grants each role to the other subjects. That is, $s_{0} \succeq_{R_{i}} s_{1}, s_{0} \succeq_{R_{i}} s_{2}$, and $s_{1} \|_{R_{i}} s_{2}$ for every role $R_{i}(i=1, \ldots, 5)$. The roles are ordered as $R_{1} \succeq R_{2} \succeq R_{3}, R_{1} \succeq R_{4} \succeq R_{5}, R_{2} \equiv R_{4}, R_{2}$ $\equiv R_{5}, R_{3} \equiv R_{4}$, and $R_{3} \equiv R_{5}$. A transaction issues five methods randomly selected from the ten methods of the object. A role is also ran-

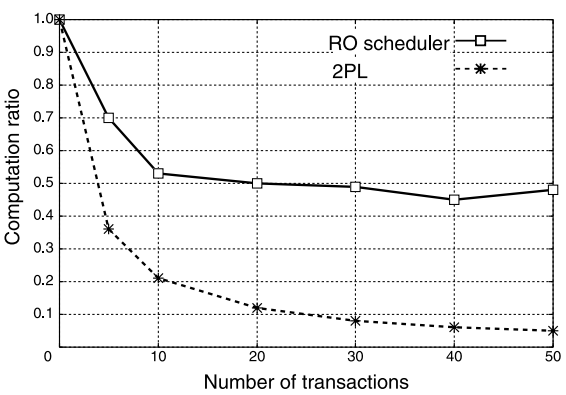

Fig. 4 Evaluation of one-object model.

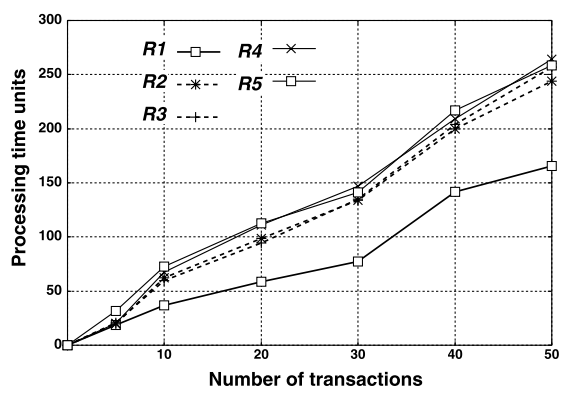

Fig. 5 RO scheduler.

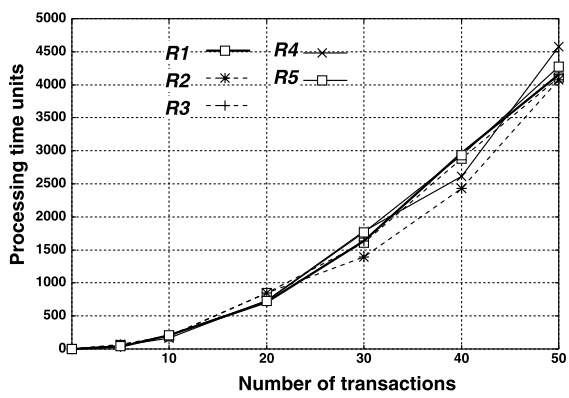

Fig. 6 Two-phase locking (2PL) protocol.

domly assigned to each transaction. The computation ratio $\tau$ is calculated multiple times in the simulation until the average value of the computation ratio is saturated.

Figure 4 shows the computation ratio $\tau$ for the number of transactions. The RO scheduler implies higher throughput than the 2PL protocol. Figures $\mathbf{5}$ and $\mathbf{6}$ show average values of processing time of the RO scheduler and the 2PL protocol, respectively, for the total number of transactions. The processing time shows the duration [time unit] from time when a method in each transaction assigned with a role $R_{i}(i$ $=1, \ldots, 5)$ is issued to time when the method completes. In the RO scheduler, a transaction $T_{i}$ which is assigned with a more significant role can manipulate an object $o$ earlier than transactions with less significant roles. On the other 
hand, the computation order of transactions is independent of the significancy of roles in the 2PL protocol.

\section{Concluding Remarks}

A role concept is widely used to design and implement information systems. The role concept shows a job function in an enterprise. In this paper, we discussed a concurrency control based on the significancy of roles assigned to transactions. We proposed a role ordering (RO) scheduler which serializes multiple conflicting transactions according to the significantly dominant relation of roles We discussed the RO scheduler for single-server and multiserver models and how to implement the RO scheduler. We showed the effectiveness of RO scheduler in terms of throughput and waiting time through the evaluation of RO scheduler compared with the traditional two-phase locking protocol $(2 \mathrm{PL})$.

\section{References}

1) Bernstein, P.A., Hadzilacos, V. and Goodman, N.: Concurrency Control and Recovery in Database Systems, Addison-Wesley (1987).

2) Bertino, E., Samarati, P. and Jaodia, S.: High Assurance Discretionary Access Control in Object Bases, Proc. 1st ACM Conf. on Computers and Communication Security, pp.140-150 (1993).

3) Chon, R., Enokido, T. and Takizawa, M.: Inter-Role Information Flow in Object-based Systems, Proc. IEEE 18th International Conf. on Advanced Information Networking and Applications (AINA-2004), Vol.1, pp.196-201 (2004).

4) Denning, D.E.: A Lattice Model of Secure Information Flow, Comm. ACM, Vol.19, No.5, pp.236-343 (1976).

5) Denning, D.E. and Denning, P.J.: Cryptography and Data Security, Addison-Wesley Publishing Company (1982).

6) Ferraiolo, D. and Kuhn, R.: Role-Based Access Controls, Proc. 15th NIST-NCSC National Computer Security Conf., pp.554-563 (1992).

7) Gray, J.: Notes on Database Operating Systems, Lecture Notes in Computer Science, No.60, pp.393-481 (1978).

8) Inc., O.M.G.: The Common Object Request Broker : Architecture and Specification, Rev. 2.1 (1997).

9) Izaki, K., Tanaka, K. and Takizawa, M.: Information Flow Control in Role-Based Model for Distributed Objects, Proc. IEEE International Conf. on Parallel and Distributed Sys- tems (ICPADS-2001), pp.363-370 (2001).

10) Oracle Corporation: Oracle8i Concepts Vol. 1 (1999). Release 8.1.5.

11) Sandhu, R.S.: Lattice-Based Access Control Models, IEEE Computer, Vol.26, No.11, pp.919 (1993).

12) Sandhu, R.S., Coyne, E.J., Feinstein, H.L. and Youman, C.E.: Role-Based Access Control Models, IEEE Computer, Vol.29, No.2, pp.3847 (1996).

13) Sybase: Sybase SQL Server. http://www.sybase.com/

14) Tachikawa, T., Yasuda, M. and Takizawa, M.: A Purpose-oriented Access Control Model in Object-based Systems, Trans. IPSJ, Vol.38, No.11, pp.2362-2369 (1997).

15) Tari, Z. and Chan, S.W.: A Role-Based Access Control for Intranet Security, IEEE Internet Computing, Vol.1, pp.24-34 (1997).

(Received April 22, 2005)

(Accepted September 2, 2005)

(Released December 7, 2005)

\section{Editor's Recommendation}

The author proposes a scheduling method based on role for distributed objects. In a sense, role may be similar to priority. They are useful to schedule objects in a suited order. The author also compares the performance of the proposed method with a conventional method and shows that the performance is clearly improved.

(Program chair of DPSWS12 Minoru Uehara)

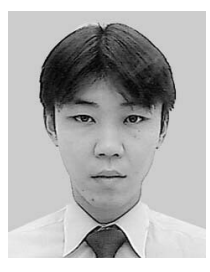

Tomoya Enokido was born in 1974. He received B.E. and M.E. degrees in Computers and Systems Engineering from Tokyo Denki University, Japan in 1997 and 1999. After that he worked for NTT Data Corporation, he joined Tokyo Denki University in 2002. He received his D.E. degree in Computer Science from Tokyo Denki University in 2003. He is currently a lecturer in the Faculty of Business Administration, Rissho University. His research interests include distributed systems, group communication, and distributed objects. 